\title{
Compared genomics of the strand switch region of Leishmania chromosome I reveal a novel genus-specific gene and conserved structural features and sequence motifs Jacques Puechberty ${ }^{1,2}$, Christine Blaineau ${ }^{1}$, Sabrina Meghamla1, Lucien Crobu ${ }^{1}$, Michel Pagès ${ }^{1}$ and Patrick Bastien*1
}

Address: ${ }^{1}$ CNRS/Université Montpellier I FRE 3013 "Biologie Moléculaire, Biologie Cellulaire et Biodiversité des Protozoaires Parasites", Laboratoire de Parasitologie-Mycologie, UFR Médecine, 163 Rue Auguste Broussonet, 34090 Montpellier, France and ²Service de Génétique Médicale, Centre Hospitalier Universitaire, Montpellier, France

Email: Jacques Puechberty - jacques.puechberty@igh.cnrs.fr; Christine Blaineau - christine.blaineau@univ-montp1.fr;

Sabrina Meghamla - meghamla_sabrina@hotmail.fr; Lucien Crobu - salulelilo@club-internet.fr; Michel Pagès - genpara@univ-montp1.fr; Patrick Bastien* - gpp@univ-montp1.fr

* Corresponding author

Published: 24 February 2007

BMC Genomics 2007, 8:57 doi:10.1186/147|-2164-8-57

This article is available from: http://www.biomedcentral.com/I47/-2164/8/57

This is an Open Access article distributed under the terms of the Creative Commons Attribution License (http://creativecommons.org/licenses/by/2.0), which permits unrestricted use, distribution, and reproduction in any medium, provided the original work is properly cited.

\begin{abstract}
Background: Trypanosomatids exhibit a unique gene organization into large directional gene clusters (DGCs) in opposite directions. The transcription "strand switch region" (SSR) separating the two large DGCs that constitute chromosome I of Leishmania major has been the subject of several studies and speculations. Thus, it has been suspected of being the single replication origin of the chromosome, the transcription initiation site for both DGCs or even a centromere. Here, we have used an inter-species compared genomics approach on this locus in order to try to identify conserved features or motifs indicative of a putative function.
\end{abstract}

Results: We isolated, and compared the structure and nucleotide sequence of, this SSR in 15 widely divergent species of Leishmania and Sauroleishmania. As regards its intrachromosomal position, size and AT content, the general structure of this SSR appears extremely stable among species, which is another demonstration of the remarkable structural stability of these genomes at the evolutionary level. Sequence alignments showed several interesting features. Overall, only $30 \%$ of nucleotide positions were conserved in the SSR among the 15 species, versus $74 \%$ and $62 \%$ in the 5' parts of the adjacent XPP and PAXP genes, respectively. However, nucleotide divergences were not distributed homogeneously along this sequence. Thus, a central fragment of approximately 440 bp exhibited $54 \%$ of identity among the 15 species. This fragment actually represents a new Leishmania-specific CDS of unknown function which had been overlooked since the annotation of this chromosome. The encoded protein comprises two trans-membrane domains and is classified in the "structural protein" GO category. We cloned this novel gene and expressed it as a recombinant green fluorescent protein-fused version, which showed its localisation to the endoplasmic reticulum. The whole of these data shorten the actual SSR to an 887 -bp segment as compared with the original $1.6 \mathrm{~kb}$. In the rest of the SSR, the percentage of identity was much lower, around $22 \%$. Interestingly, the 72 -bp fragment where the putatively single transcription initiation site of chromosome I was identified is located in a low-conservation portion 
of the SSR and is itself highly polymorphic amongst species. Nevertheless, it is highly C-rich and presents a unique poly $(\mathrm{C})$ tract in the same position in all species.

Conclusion: This inter-specific comparative study, the first of its kind, (a) allowed to reveal a novel genus-specific gene and (b) identified a conserved poly $(\mathrm{C})$ tract in the otherwise highly polymorphic region containing the putative transcription initiation site. This allows hypothesising an intervention of poly $(\mathrm{C})$-binding proteins known elsewhere to be involved in transcriptional control.

\section{Background}

The protozoan parasite Leishmania $s p$. is responsible for significant worldwide human morbidity and mortality, and the clinical features of diseases vary depending on the causative species. The genus Leishmania belongs to the family of trypanosomatids with, among others, Trypanosoma brucei and T. cruzi, responsible for sleeping sickness and Chagas' disease, respectively. Taxonomically, it is separated into two sub-genera:L. (Leishmania) and L. (Viannia). The differentiation between both appears very ancient, as it is estimated to be contemporary to the formation of the Gondwana, i.e. around 60 million years [1]. However, comparative genomics showed the general structure of the genome to be remarkably stable within this genus. Chromosomal synteny groups are entirely conserved for all Old World species (subgenus L. (Leishmania)) where 36 heterologous chromosomes have been identified [2]. As regards the New World, only two and one large chromosomal rearrangements, according to the subgenus, have been shown as compared with Old World species. This leads to a chromosome number of 34 and 35 for L. (Leishmania) and L. (Viannia) species, respectively, but with all Old World species linkage groups remaining conserved [3]. Similarly, chromosomal restriction maps showed a complete conservation of collinearity of markers between species [4]. Finally, the sequencing of the 'TriTryp' genomes also demonstrated a high degree of synteny among those three protozoa (L. major, T. brucei and $T$. cruzi) [5].

Trypanosomatids exhibit a number of highly original molecular and cellular biological features. Among those, one may cite systematic trans-splicing, consisting in the addition of a 39 nt-spliced leader RNA at the 5'end of all mRNAs, and the near absence of promoters for polymerase II, implying an absence of regulation of gene expression at the transcriptional level. One of the most extraordinary features revealed by these genome projects was the gene organisation into large collinear clusters present on a single strand and comparable to prokaryotic polycistronic units, except that the genes present have no common nor akin function [6-8]. These large directional gene clusters (DGCs) are separated by short sequences of a few kbs termed coding strand switches or strand-switch regions (SSRs), where the transcription sense converges or diverges. This remarkable position led several authors to express different hypotheses upon the putative function of these regions in Leishmania.

The comparative in silico analysis of several SSRs in $L$. major only revealed low homologies at the structural as well as nucleotide sequence level $[5,8,9]$ that render difficult a common interpretation as for their putative role. AT distribution and hairpin content analysis failed revealing common features to different SSRs but allowed separating them into two groups with respect to the adjacent gene clusters transcription orientation (divergent or convergent) [9]. The same authors showed that the five SSRs analysed presented a very high intrinsic DNA curvature, the latter being classically associated with transcription as well as replication and centromere functions (reviewed in [7]).

However, experimental data showed that the deletion of the sole SSR of chromosome 1 did not affect mitotic stability, hence it was not necessary for chromosomal replication nor segregation [10]. This goes against the hypotheses of this SSR being a single replication origin [11] or a centromere [9]. It is noteworthy that, whereas in T. cruzi, a 16-kb SSR, made for a large part of retroelements, has been identified as a centromere [12], in Leishmania, the centromeric function on chromosome 1 could be attributed to a subtelomeric 20 -kb satellite repeat cluster [13]. On the other hand, run-on experiments showed that this same SSR on L. major chromosome 1 contained a single site of bi-directional initiation of transcription for both gene clusters [14]. This might explain why the deletion of this SSR could not be realised on the three copies of chromosome 1 in the reference strain L. major 'Friedlin' [10]. On the other hand, the expression of a reporter gene inserted into one of both gene clusters was not affected by the deletion of the SSR [10], which shows that transcription is possible without the presence of the SSR. Myler et al. explained the latter fact by a minor 'residual' level of expression on the chromosome that would not initiate at the SSR itself [14]: still, this level of transcription is sufficiently high to allow the expression of the reporter protein.

Here, we bring new elements in the knowledge of the structure and conservation of these regions using an infrageneric compared genomics approach. We have 
sequenced the SSR of chromosome 1 in 15 highly divergent species of Leishmania and Sauroleishmania (of which the inclusion within Leishmania remains uncertain) [15] and show the presence of conserved structural elements and motifs that were overlooked during sequence annotation or using inter-genera comparative genomics.

\section{Results and discussion The general structure of the "switch region" is conserved in all Leishmania species}

We have analyzed the SSR of chromosome 1 in 15 species of Leishmania and Sauroleishmania. In each species, this region was amplified by using PCR primers located on the 5 ' part of the two genes adjacent to the SSR in L. major: PAXP and XPP (Fig. 1). For all species, a fragment of expected size (approximately $1650 \mathrm{bp}$ ) was found. This fragment was cloned and sequenced [GenBank ${ }^{\mathrm{TM}}$ accession numbers DQ522034 to DQ522048]: this confirmed the presence of both genes adjacent to the SSR. As expected, the transcription direction of both genes is in opposite sense and directed towards the telomeres in all species. The distance between the start codons of both genes is relatively constant as it lies between 1640 and 1669 bp (for L. aethiopica and L. amazonensis respectively) (Table 1 ). Incidentally, our data allow repositoning the start codon of the PAXP gene at position 79049 instead of 79145. The SSR is thus remarkably size-stable since it varies of $<2 \%$ among both extremes. These results support previous studies on the conservation of chromosomal linkage groups $[2,3]$, or the compared mapping of certain chromosomes in various species [4], showing a surprising interspecific conservation of the organization of these genomes. As described in L. major [6], the AT content of this sequence in the various species is relatively high since it ranges between 50\% (L. enriettii) and 54\% (Sauroleishmania) (Table 1). This relative AT-richness distinguishes the SSR from the remainder of the genome where it is 35\% [6]. This physical characteristic may facilitate weaker binding of the two strands during either transcription or replication processes, and is classically found in the noncoding regions with a structural role such as centromeres or replication origins $[16,17]$. The whole of these features shows that the general structure of this region is highly conserved among the 15 species studied here.

\section{Presence of a conserved CDS in the switch region}

An alignment of the 15 sequences amplified as above was realised using ClustalW software. Figure 2 represents the rates of conserved nucleotides (nt) among the 15 species studied, at the level of the SSR on the one hand, and of the adjacent genes on the other hand. The 5' part of both genes is highly conserved, with a mean identity rate among all 15 species of $74 \%$ and $62 \%$ for XPP and PAXP respectively (analysed on 97 and 109 bp, respectively); most mutations being silent, this yields identity rates at

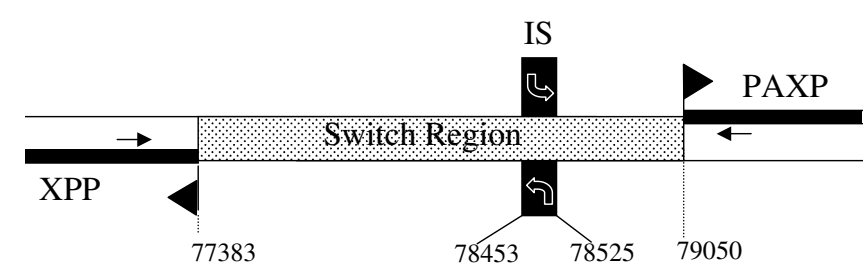

Figure I

Schematic representation of the strand switch region (SSR) of chromosome I in L. major Friedlin. The nt positions (figures) refer to the complete sequence of this chromosome available on the GeneDB website [28]. XPP: acidocalcisomal exopolyphosphatase gene; PAXP: poly(A) export protein gene; IS: putative transcription initiation site, with the curved arrow showing transcription sense [14]. The start codons of both genes are indicated by a closed arrowhead. It is noteworthy that, after careful compared analysis with the genomes of Trypanosoma brucei and T. cruzi, we repositioned the start codon of PAXP at position 79049 and not at 79/45 as wrongly indicated on GeneDB. The positions of the primers on the XPP and PAXP genes are indicated as arrows.

the amino acid level of $80 \%$ and $78 \%$ respectively. It should be noted that the comparison of 100 bps from a Leishmania gene generally yields divergence rates representative of those obtained when one compares the whole length of the gene (see Legend of Fig. 2). At the level of the SSR, the overall nt divergence appears much more significant: only $30 \%$ of nt positions are conserved among the 15 sequences. Interestingly, this divergence is not distributed homogeneously along this sequence. Thus, a central fragment of approximately 440 bp (429 bp in L. major), termed B, exhibits $54 \%$ of identity among the 15 species (Table 1 and Fig. 2). It contrasts with most of the rest of the SSR (except fragment D, see below) where this percentage is much lower, being $23 \%$ and $22 \%$ for fragment A (left) and $\mathrm{C}$ (right), respectively. The quality of the alignment of fragment $\mathrm{B}$ is actually close to that observed in the 5' parts of the XPP and PAXP genes (Fig. 2). A closer analysis revealed that it actually corresponds to an ORF conserved in the whole of the species, hence to a CDS [see Additional file 1: Amino acid sequence alignment], whose transcription orientation would be towards the 'left' of the chromosome. BLAST analysis did not show any valid homology of this sequence to any other SSR not to any other organism, in particular in the genomes of T. brucei 
Table I: Comparative features of the chromosome I strand switch region and the 5 '-ends of the adjacent XPP and PAXP genes in I5 Leishmania species ${ }^{a}$.

\begin{tabular}{|c|c|c|c|c|c|c|c|}
\hline \multirow[t]{2}{*}{ Species } & \multicolumn{2}{|c|}{$\mathbf{X P P}$} & \multicolumn{3}{|c|}{ Switch region } & \multicolumn{2}{|c|}{ PAXP } \\
\hline & $\%$ nt div. ${ }^{b}$ & $\operatorname{div} . \mathrm{aa} / 32 \mathrm{aa}^{\mathrm{c}}$ & Size (bp) & $\%$ AT & $\% n t \operatorname{div} d$ & $\%$ nt div. ${ }^{e}$ & div. aa/36aa ${ }^{f}$ \\
\hline L. aethiopica & 1 & I & 1640 & 51 & 15 & I & 0 \\
\hline L. arabica & i & 0 & 1658 & 50 & 16 & 4 & I \\
\hline L. turanica & 2 & 1 & 1665 & 50 & 22 & 2 & 0 \\
\hline L. major & 3 & 0 & 1666 & 50 & 16 & 5 & 1 \\
\hline L. tropica & 2 & 0 & 1659 & 50 & 14 & 2 & I \\
\hline L. archibaldi & 1 & 0 & 1668 & 51 & 16 & 3 & I \\
\hline L. donovani & I & 0 & 1666 & 50 & 14 & 2 & I \\
\hline L. infantum & 1 & 0 & 1662 & 50 & 15 & 3 & I \\
\hline L. amazonensis & 3 & 0 & 1669 & 52 & 16 & 2 & 0 \\
\hline Sauroleishmania & 9 & 3 & 1643 & 54 & 25 & 4 & 0 \\
\hline L. peruviania & 3 & I & 1656 & 53 & 23 & 13 & 4 \\
\hline L. braziliensis & 3 & I & 1657 & 53 & 24 & 10 & 3 \\
\hline L. guyanensis & 3 & I & 1652 & 53 & 23 & 11 & 3 \\
\hline L. sp. MARI & 12 & 4 & 1658 & 51 & 30 & 12 & 0 \\
\hline L. enriettii & 6 & I & 1642 & 50 & 25 & 8 & 0 \\
\hline
\end{tabular}

${ }^{a}$ For each species, the divergent nucleotides or amino acids were counted as compared with the consensus sequence; ${ }^{b}$ Percentage of nucleotide (nt) divergence established from the sequence of $97 \mathrm{nt}$ at the $5^{\prime}$ end of the gene; all percentages were rounded up to the closest integer number; ${ }^{c}$ Number of unconserved amino acids (aa) out of 32 aa at the N-terminal end of the protein; ${ }^{d}$ Percentage of nucleotide (nt) divergence established from the consensus sequence over the whole SSR as defined in the Material and Methods; e Percentage of nucleotide (nt) divergence established from the sequence of $109 \mathrm{nt}$ at the $5^{\prime}$ end of the gene; ${ }^{\mathrm{f}}$ Number of unconserved amino acids (aa) out of 36 aa at the N-terminal end of the protein.

and T. cruzi. This CDS, with no putative function, was recently added in the L. major GeneDB database.

Another moderately conserved fragment, termed D, was also noted in the ca. 120 nucleotides upstream of the PAXP gene (Fig. 2) in the SSR. Of note on this fragment is the putative spliced leader RNA addition site (AG) for PAXP [14] which is conserved in all 15 species. By contrast, it is noteworthy that the putative trans-splicing acceptor site (SAS) of the XPP gene, that was identified in the same report 840 bp upstream of the methionine codon [14], is not conserved amongst all species (only in $9 / 15$ species) and is now located $50 \mathrm{nt}$ upstream of the newly identified CDS, hence was probably misidentified. The putative conserved SAS of the new CDS is likely located $20 \mathrm{nt}$ upstream of its start codon. The sequence alignment allows the identification of only one candidate SAS for the XPP gene that would be conserved amongst all Leishmania species (but not Sauroleishmania), $50 \mathrm{nt}$ upstream (L. major sequence) of the start codon of XPP.

\section{Analysis of the Leishmania-specific CDS}

As it is is not conserved among the Tri-Tryps (L. major, T. brucei and T. cruzi), this CDS therefore appears Leishmania-specific. As such, it constitutes one example of a species-specific gene occurring at a synteny breakpoint between these three organisms, since the SSR analysed here forms such a breakpoint [5]. In T. cruzi, this SSR is conserved as such (1211 bp, with the XPP and PAXP gene located on the 'left' and 'right' of the SSR respectively); but another unknown CDS is located upstream of the PAXP gene, hence at the start of the 'right-oriented' directional gene cluster (DGC) (like our novel gene is located at the start of the 'left-oriented' DGC in Leishmania). This CDS in $T$. cruzi is conserved in the same location and DGC in $T$. brucei, suggesting that our novel gene likely is the result of both a gene deletion (that of the CDS of T. cruzi and T. brucei) and a gene insertion (that of our novel CDS) events during evolution. By contrast, the region is not conserved as such in T. brucei, where it sizes $8286 \mathrm{bp}$, bears several 'unlikely' CDSs and retroelements and, more importantly, does not actually constitute a SSR; still, it is flanked downstream by the same DGC as in the other Tri-Tryps and upstream by the upstream DGC of L. major chromosome 1 but entirely inverted [5].

The function of this new gene is unknown (like $>60 \%$ of the Leishmania genes). The Protfun program allowed predicting its putative function in either energy metabolism or cell enveloppe, and classified it in the "structural protein" GO category. Further bioinformatic analysis showed that the encoded 143 amino acid protein (in L. major) comprises two transmembrane helices (residues 32-54 and 104-126; probablity = 0.99 using TMHMM and InterProScan software). As regards post-transcriptional processing, a signal peptide was identified at the N-terminus (according to Signal-P); and two serine and one threonine are potential phosphorylation sites (according to NetPhos). Finally, TargetP shows a strong prediction for a mitochondrial localisation, but targeting signals are often 


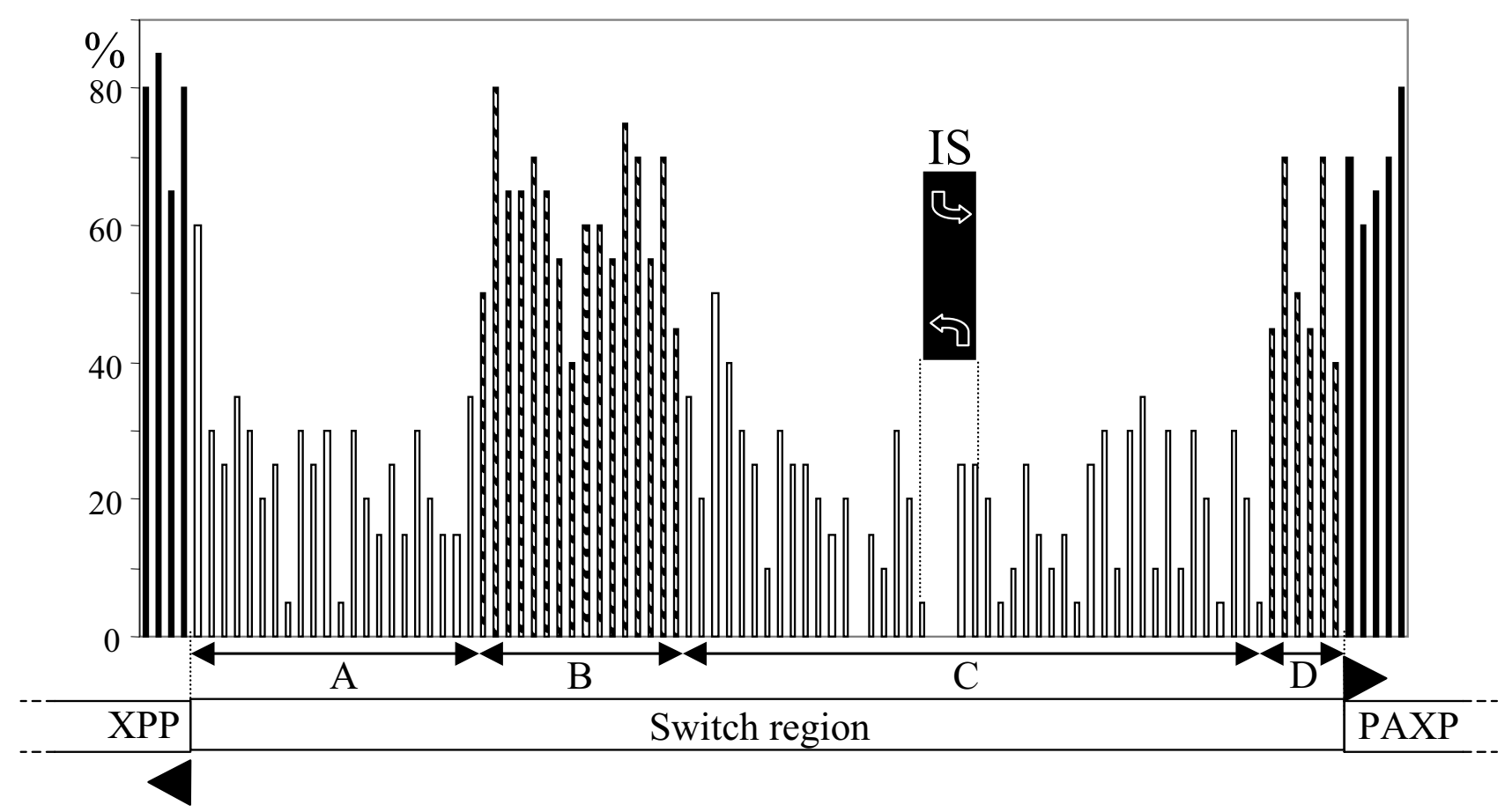

\section{Figure 2}

Histogram representation of the rates of identical nt positions amongst the SSR sequences of I5 Leishmania species. Each vertical bar represents a $20 \mathrm{nt}$ window. At each end, 109 and $97 \mathrm{bP}$ cover the $5^{\prime}$ ends of PAXP and XPP genes, respectively (black bars). Two segments of the SSR appear highly conserved ( $B$ and $D$, hatched bars) as compared with other segments (A and C, open bars). It is noteworthy that the comparison of 100 bps from a Leishmania gene generally yields divergence rates similar to those obtained when one compares the whole length of the gene : e.g. here, for PAXP, the identity rates between the sequences from $L$. major and $L$. braziliensis are $83.5 \%$ for the whole gene (now available in GeneDB) and $85.7 \%$ for the 109 bps analysed here. XPP, PAXP, IS as in Fig. I.

non-conserved in Trypanosomatids [[18]; our unpublished data].

We then constructed an episomal vector expressing a recombinant green fluorescent protein (GFP)-fused version of the protein after transfection in L. major, in order to observe its subcellular localisation. Combined cell staining wih Mitotracker showed that, in opposition with the predictions of TargetP, the protein is not addressed to the mitochondrion (Fig. 3). By contrast, it localises to a subpellicular, cytoplasmic and perinuclear network, that is clearly not overlapping the mitochondrion, most likely the endoplasmic reticulum. This localisation is compatible with the presence of two transmembrane domains. It is noteworthy that no phenotype (cell growth, cell cycle, morphology) could be associated with the episomal overexpression of the protein (not shown).

The putative transcription initiation site is located in a highly variable segment

Martinez-Calvillo et al. [14] identified a 73-bp segment on the SSR as the putative transcription initiation site on chromosome 1 (position 78453-78525). Fig. 4 presents the inter-specific alignment of the nt sequences corresponding to this segment. Interestingly enough, this sequence is located in the portion of the SSR with the highest inter-specific nt divergence (IS in Fig. 2). Moreover, the sequence itself is highly polymorphic amongst the various species (Fig. 4). However, this segment shows notable conserved features in all species: (i) as noted previously in L. major [14], it has the highest GC rate (ca. $70 \%$ ) in the whole SSR, and particularly is highly C-rich; (ii) it presents a unique poly $(\mathrm{C})$ tract conserved in the same position, although of variable length, in all species, including in highly divergent species like L. enriettii or MAR1 (see below). The presence of this poly $(\mathrm{C})$ tract was not specifically mentioned in the corresponding paper [14]. Although it may be considered as a simple polypyrimidine tract, it is the only conserved tract in the whole segment, and this conserved feature makes it is tempting to speculate upon its possible role. The high C-content may here induce a conformation of the DNA double helix supporting RNA polymerase entry. Moreover, poly(C)binding proteins, a conserved subfamily of K-homology 

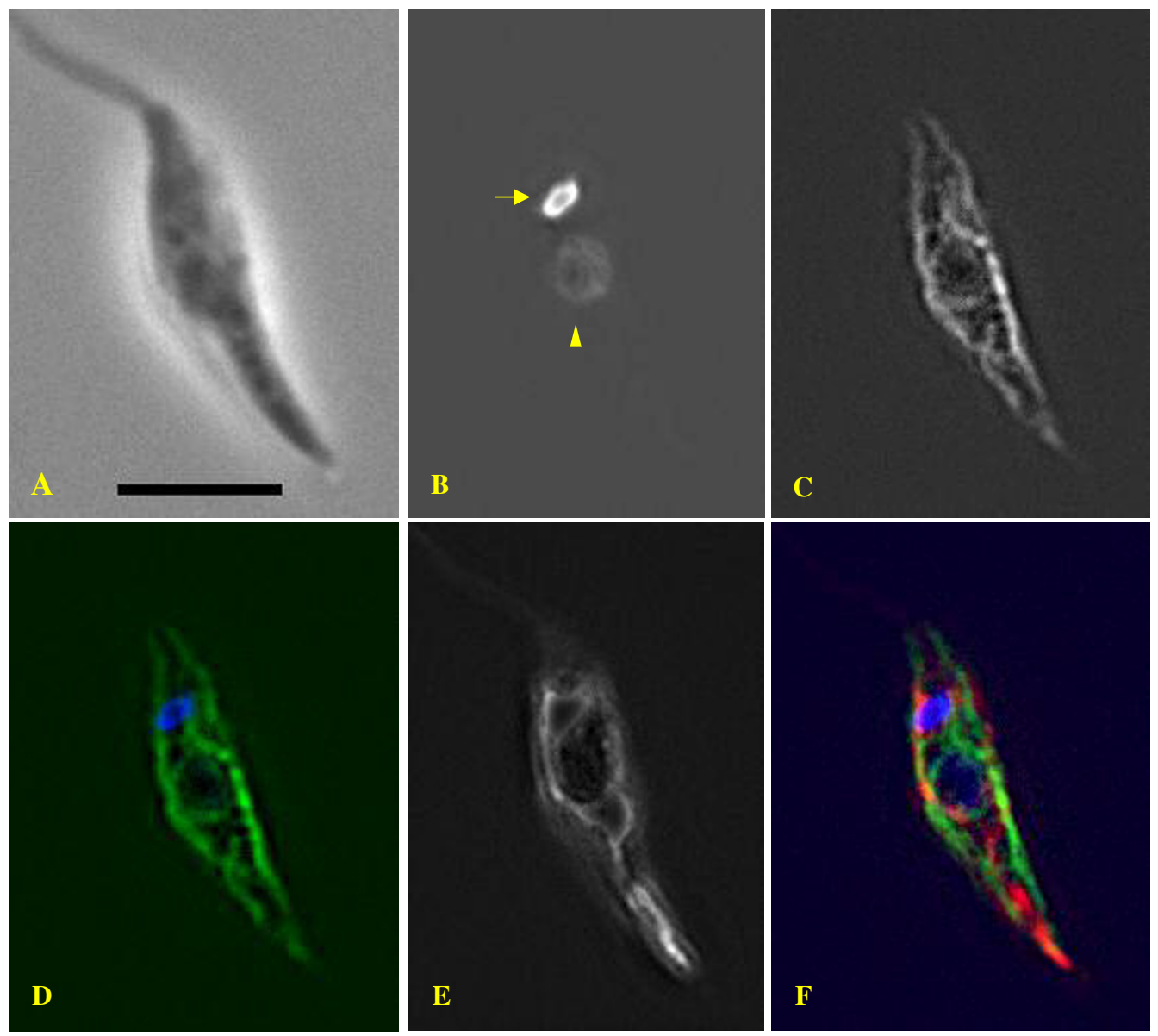

\section{Figure 3}

The protein encoded by the novel Leishmania-specific gene localises to the endoplasmic reticulum. Images of an L. major 'Friedlin' promastigote expressing a recombinant GFP-fused version of the protein encoded by the novel CDS. (A) Phase contrast microscopy; scale bar : 10 microns. (B) DAPI-staining of the nucleus (arrowhead) and the single mitochondrial DNA or kinetoplast (arrow). (C) Localisation of the GFP-fused protein viewed in fluorescence. (D) Colour combination of GFP (green) and DAPI (blue) fluorescence. (E) Mitochondrion labeled with Mitotracker ${ }^{\mathrm{TM}}$. (F) Merged picture: blue = DNA; red $=$ Mitotracker ${ }^{\mathrm{TM}}$; green = GFP-fused protein. The protein localised to a subpellicular, cytoplasmic and perinuclear network that is clearly different from the mitochondrion and closely resembles the endoplasmic reticulum; the latter being identified by expression of the plasmid construct GFP-MDDL that acts as an endoplasmic reticulum retention signal in trypanosomatids [3 I] [see Additional File 2].

domain-containing proteins, are known, among other functions, to be involved in transcriptional control through a variety of mechanisms [19] and in particular to activate transcription of the human $c-m y c$ gene [20].

\section{The switch region sequences alignment yields a coherent taxonomic tree}

The phylogenetic tree built from these SSR sequences clearly identified the various groups classically defined in the genus Leishmania (Fig. 5) [15]. Thus, a very high level of homology can be observed within groups that comprise classically very closely related species such as $L$. major-L. arabica-L. turanica and L. peruviana-L. brasiliensis-
L. guyanensis, with $98.8 \%$ and $97.6 \%$ of identical nucleotide positions over the whole SSR, respectively. Between these two groups, which may be taken as representatives of the L. (Leishmania) and L. (Viannia) subgenera respectively, the percentage of identity is only $69 \%$, supporting the current taxonomic dichotomy. A homology rate of $83.7 \%$ for this SSR had previously been reported among L. major, L. donovani, L. infantum, L. mexicana and L. amazonensis [14]. Interestingly, two species are shown clearly differentiated at the basis of the tree: L. sp. MAR1 and $L$. enriettii. The first one corresponds to extremely rare isolates from cutaneous leishmaniasis patients from the Caribbean [21]. The second one is an animal species isolated 


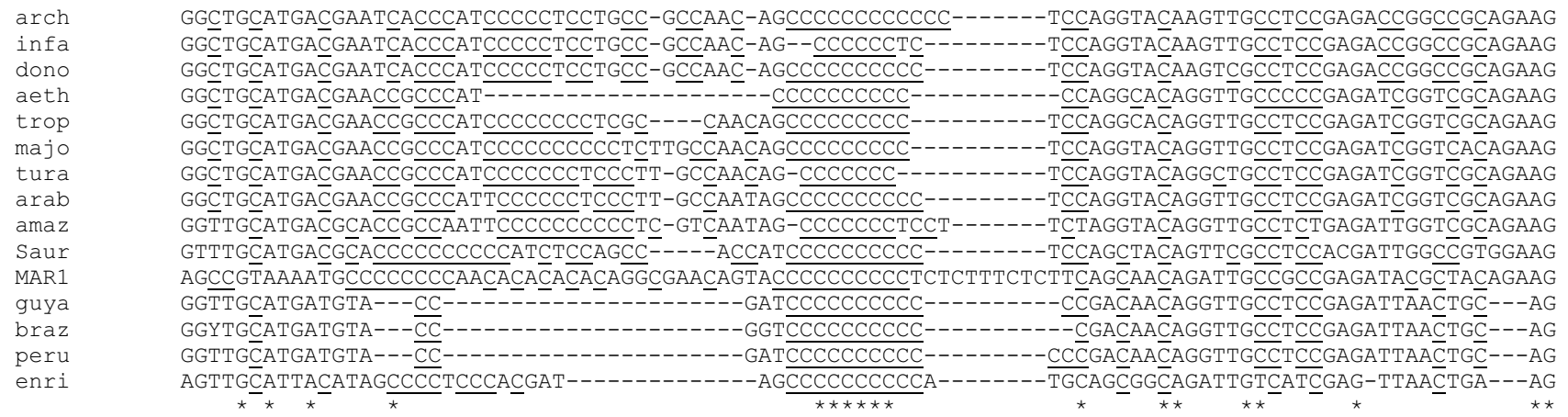

\section{Figure 4}

Alignment of the sequences corresponding to the putative bidirectional transcription initiation site among the I 5 Leishmania species analysed. Alignment of the sequences corresponding to the putative bidirectional transcription initiation site [14] among the 15 Leishmania species analysed. This shows the presence of a unique and conserved poly(C) in all species studied here. Cytidines are shown underlined. Asterisks indicate the nucleotides conserved in all species. The first four letters of each line indicate the species name (see Table I).

on rare occasions from the guinea-pig [22] and was found as the most external member of the genus Leishmania [23]. Both species also clustered together at a basal position for all other Euleishmania by molecular phylogeny using DNA polymerase alpha and RNA polymerase II geneencoding sequences [15]. Finally, it is noteworthy that these sequence alignments strongly support the inclusion of Sauroleishmania (of which the taxonomic position remains controversial; reviewed in [1]) within Leishmania and perhaps in an intermediary position between both subgenera.

\section{Conclusion}

This study is the first of its kind in Trypanosomatids as it is based on an 'inter-specific' study (comparing 15 Leishmania species), as opposed to the vast analysis that had been published in 2005 that compared the "Tri-Tryps", $L$. major, T. brucei and T. cruzi [5]. The interest of the first approach is to identify Leishmania-specific genes that would have been overlooked in the second approach, e.g. this new CDS. This is a novel demonstration of the interest of a (here infrageneric) compared genomics approach in identifying unknown genes or functional motifs. The presence of this CDS might partly explain the difficulties encountered in knocking-out the SSR [10], if this endoplasmic reticulum-restricted Leishmania-specific gene had an essential role.

Considering its transcription sense, these data also actually shortens the 'proper' strand switch region to an 877 bp segment (segment C in Fig. 2, position 78173 to 79049 on L. major Friedlin chromosome 1).

This study also sheds new light upon the putative function of the SSR of chromosome 1. Surprisingly indeed, the putative transcription initiation site (TIS) previously identified on this chromosome by run-on analysis [14] is found in the most polymorphic portion of the SSR; yet, it presents a conserved poly $(\mathrm{C})$ tract in a highly conserved position in divergent Leishmania species. These data both question and reinforce the TIS hypothesis. The high nt sequence polymorphism of this segment and the ubiquitous presence of poly $(\mathrm{C})$ tracts make it difficult to define structural features of TISs in Leishmania. Conversely, the fact that a number of genes encoding K-homology domain-containing proteins have been identified within the Leishmania genome make it possible to hypothesize that these proteins participate in transcriptional control. Experimental analysis of these genes may help in understanding transcription initation mechanisms used by this unusual parasite.

\section{Methods}

\section{Parasite strains}

The Leishmania strains used for sequencing the switch region of chromosome 1 were: in the sub-genus L. (Leishmania), $L$. donovani LEM138 (MHOM/IN/00/DEVI), $L$. infantum LEM1136 (MHOM/FR/87/LEM1136-cl), $L$. archibaldi LEM1005 (MHOM/ET/72/GEBRE1), L. major LEM134 (MHOM/SU/73/5-ASKH), L. major 'Friedlin' (MHOM/IL/81/FRIEDLIN), L. turanica LEM558 (MRHO/ SU/74/95A), L. arabica LEM1108(MPSA/SA/83/JISH220), L. aethiopica LEM1660 (MHOM/ET/89/LEM1660-CL), L. tropica LEM84 (MRAT/IQ/72/ADHANIS1), L. amazonensis LEM2246 (IFLA/TT/71/71-110), L. enriettii LEM1120 (MCAV/BR/45/L88) and L. sp. MAR1 or LEM2494 (MHOM/MQ/92/MAR1) [22]; in the sub-genus L. (Viannia), L. braziliensis LEM396 (MHOM/BR/75/M2903), $L$. peruviana LEM1535 (MHOM/PE/84/UN59) and L. guyanensis LEM85 (MHOM/GF/79/LEM85); and in the genus 


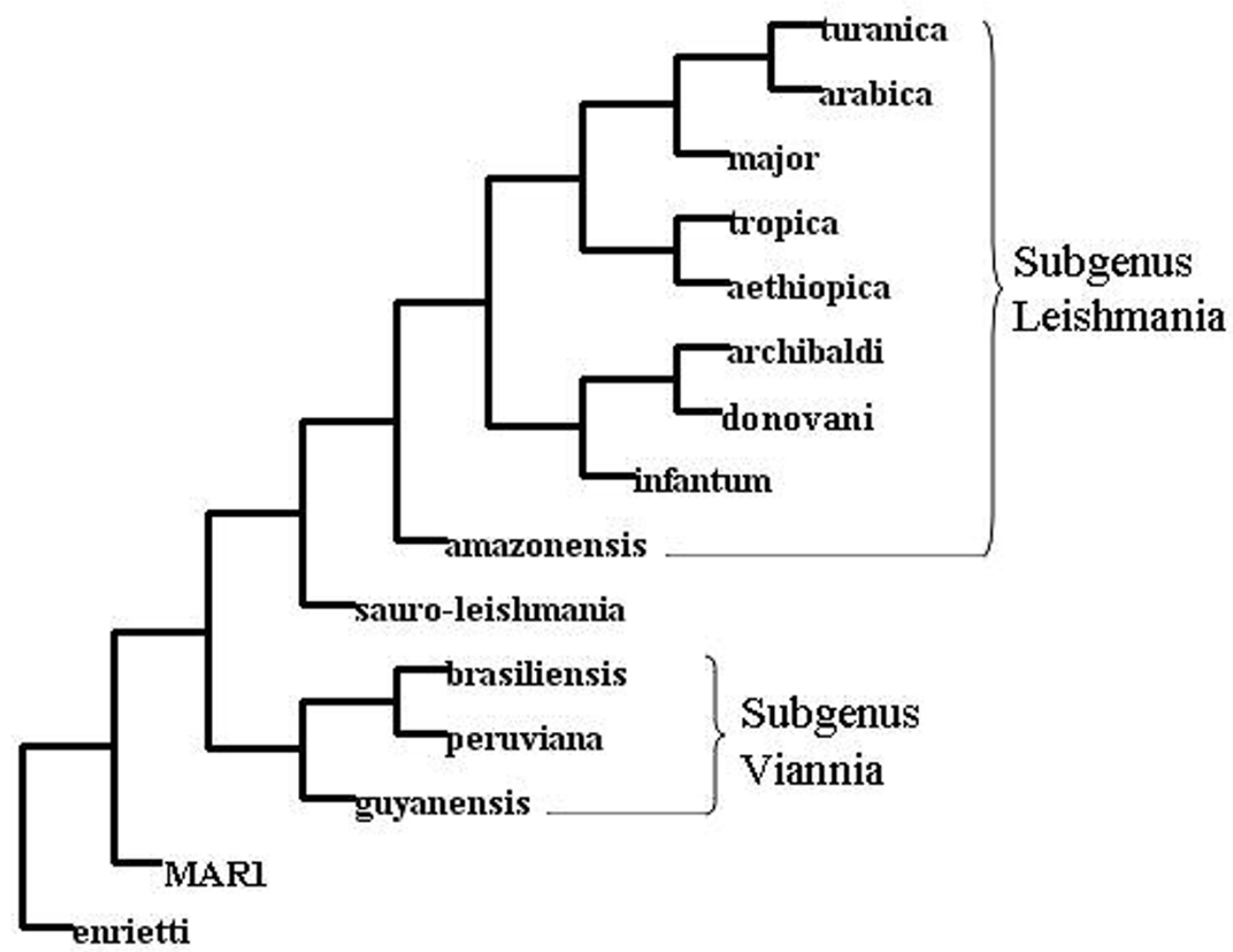

Figure 5

Phylogenetic analysis of 15 divergent Leishmania species obtained from the chromosome I strand switch region sequence alignment. Unrooted tree produced from the chromosome I strand switch region sequence alignment by the DNAMP program in DAMBE (see text for comments). Names represent different Leishmania species (see Table I).

Sauroleishmania, S. tarentolae LEM351 (RTAR/DZ/39/TARVI). All strains were cultivated on blood agar (NovyMcNeal-Nicolle) medium. Their identity was checked by examination of 15 isoenzyme systems immediately prior to this study [24].

\section{PCR conditions and sequences}

Genomic DNA extraction of each strain was performed by phenol-chloroform, followed by ethanol precipitation. All DNA regions analysed were PCR-amplified from total genomic DNA using the high -fidelity Platinum ${ }^{\circledR}$ Pfx DNA Polymerase (Invitrogen ${ }^{\circledR}$ ). Different primers were used for targeting different areas of the acidocalcisomal exopolyphosphatase $(X P P)$ gene, the poly(A) export protein $(P A X P)$ gene and the central part of the SSR: forward primers ccgacaatgctgtccatgt and gtggcaatgcaaatgggcagc; and reverse primers gcaactcccgtcccacga, and tgagcgcgcgacttgtcg. After electrophoresis, PCR products were purified from agarose gels and cloned into pGEMT-Easy (Promega $^{\circledR}$ ), then sequenced on a Licor $^{\circledR}$ automated sequencer and later assembled using the Sequencher ${ }^{\mathrm{TM}}$ software (Gene Codes Corp. ${ }^{\circledR}$ ). All sequences were double strand reads and the quality of the sequence obtained was carefully checked manually.

\section{Sequence analysis}

Nucleotide sequence alignments and phylogenetic trees were realised using ClustalW [25] and Dambe software [26]. Homologies were searched via Blast on two distinct websites [27,28]. Bioinformatic analysis of the protein was done using CBS [29] and EBI [30] software resources.

\section{Expression of the recombinant protein}

The coding region of the novel CDS, with the start and stop codons removed, was PCR-amplified from L.major 'Friedlin' genomic DNA with specific forward and reverse 
oligonucleotides containing the MfeI and HpaI restriction sites respectively. The PCR product, purified and digested with MfeI-HpaI, was cloned into the MfeI and HpaI sites of the plasmid vector $\mathrm{pTH}_{6}$ nGFPc [18], generating a construct where the CDS is fused to the GFP gene in its 3 ' end. $100 \mu \mathrm{g}$ of episomal DNA plasmid were then transfected by electroporation into $8 \times 10^{7} \mathrm{~L}$. major 'Friedlin' promastigotes grown to mid-log phase, which were grown under selection pressure with hygromycin at $30 \mu \mathrm{g} / \mathrm{ml}$. Leishmania cells were then viewed in microscopy and photographed as described [18]. The mitochondrion was visualised by incubating cultures in MitoTracker Red CMXRos (Molecular Probes ${ }^{\circledR}$ ) for 10 min prior to fixation.

\section{Authors' contributions}

JP and SM carried out the molecular genetic studies and participated in the sequence alignment. CB participated in the sequence alignment and analysis. CB and LC constructed the plasmid vectors and performed the transfection analysis. SM and MP drafted the manuscript. MP participated in the design of the study and performed the sequence data analysis. PB conceived the study, and participated in its design and coordination and helped to draft and finalize the manuscript. All authors read and approved the final manuscript.

\section{Additional material}

\section{Additional File 1}

Alignment of the amino acid sequences of the novel CDS among 15 Leishmania species. The data provided represent the alignment of the amino acid sequences of the novel CDS identified in the central part of the chromosome 1 switch region among 15 Leishmania species. Colour codes indicate groups of amino acids (small, acidic, basic...) and are explicited on the EBI website [32]. "* " residues identical in all sequences; ":" conserved substitutions; ". " semi-conserved substitutions. Species are indicated by their three first letters (see Table 1), except L. sp. MAR1 shown as "anc" (standing for "ancestral").

Click here for file

[http://www.biomedcentral.com/content/supplementary/14712164-8-57-S1.ppt]

\section{Additional File 2}

Visualisation of the endoplasmic reticulum (ER) in Leishmania major using an ER-marker. The pictures provided show an L. major cell where the ER was visualised using an ER-specific expression plasmid construct. Images of an L. major promastigote form expressing the plasmid construct GFP-MDDL that acts as an endoplasmic reticulum retention signal in trypanosomatids [31]. Upper left: phase contrast microscopy; scale bar : 10 microns. Upper right (DAPI): DAPI-staining of the nucleus and kinetoplast. Lower left (BIP-GFP): Localisation of the GFP-MDDL marker viewed in fluorescence. Lower right (MERGE): Colour combination of GFP (green) and DAPI (red) fluorescence.

Click here for file

[http://www.biomedcentral.com/content/supplementary/14712164-8-57-S2.ppt]

\section{Acknowledgements}

We wish to thank Jean-Pierre Labbé for fruitful discussions and thoughts about the putative roles of the strand switch region. We also acknowledge the technical assistance of Yves Balard, as well as Jean-Pierre Dedet for continous support. This study was funded by the CNRS and University

Montpellier I.

\section{References}

I. Momen H, Cupolillo E: Speculations on the origin and evolution of the genus Leishmania. Mem Inst Oswaldo Cruz 2000, 95:583-588.

2. Wincker $P$, Ravel $C$, Blaineau $C$, Pagès $M$, Jauffret $Y$, Dedet JP, Bastien $P$ : The Leishmania genome comprises 36 chromosomes conserved across widely divergent human pathogenic species. Nucleic Acids Res 1996, 24: 1688-1694.

3. Britto $C$, Ravel C, Bastien P, Blaineau C, Pagès M, Dedet JP, Wincker $P$ : Conserved linkage groups associated with large-scale chromosomal rearrangements between Old World and New World Leishmania genomes. Gene 1998, 222:107-I 17.

4. Ravel C, Dubessay P, Britto C, Blaineau C, Bastien P, Pagès M: High conservation of the fine-scale organisation of chromosome 5 between two pathogenic Leishmania species. Nucleic Acids Res 1999, 27:2473-2477.

5. El-Sayed NM, Myler PJ, Blandin G, Berriman M, Crabtree J, Aggarwal G, Caler E, Renauld H, Worthey EA, Hertz-Fowler C, et al.: Comparative genomics of trypanosomatid parasitic protozoa. Science 2005, 309:404-409.

6. Myler PJ, Audleman L, deVos T, Hixson G, Kiser P, Lemley C, Magness C, Rickel E, Sisk E, Sunkin S, et al:: Leishmania major Friedlin chromosome I has an unusual distribution of protein-coding genes. Proc Natl Acad Sci USA 1999, 96:2902-2906.

7. Worthey EA, Martinez-Calvillo S, Schnaufer A, Aggarwal G, Cawthra J, Fazelinia G, Fong C, Fu G, Hassebrock M, Hixson G, et al.: Leishmania major chromosome 3 contains two long convergent polycistronic gene clusters separated by a tRNA gene. Nucleic Acids Res 2003, 3 I:4201-42I0.

8. Ivens AC, Peacock CS, Worthey EA, Murphy L, Aggarwal G, Berriman M, Sisk E, Rajandream MA, Adlem E, Aert R, et al.: The genome of the kinetoplastid parasite, Leishmania major. Science 2005, 309:436-442.

9. Tosato V, Ciarloni L, Ivens AC, Rajandream MA, Barrell BG, Bruschi $C V$ : Secondary DNA structure analysis of the coding strand switch regions of five Leishmania major Friedlin chromosomes. Curr Genet 200I, 40:186-194.

10. Dubessay $P$, Ravel $C$, Bastien $P$, Crobu L, Dedet JP, Pagès M, Blaineau $C$ : The switch region on Leishmania major chromosome $I$ is not required for mitotic stability or gene expression, but appears to be essential. Nucleic Acids Res 2002, 30:3692-3697.

II. McDonagh PD, Myler PJ, Stuart KD: The unusual gene organization of Leishmania major chromosome I may reflect novel transcription processes. Nucleic Acids Res 2000, 28:2800-2803.

12. Obado SO, Taylor MC, Wilkinson SR, Bromley EV, Kelly JM: Functional mapping of a trypanosome centromere by chromosome fragmentation identifies a 16-kb GC-rich transcriptional "strand-switch" domain as a major feature. Genome Res 2005, 15:36-43.

13. Dubessay P, Ravel C, Bastien P, Stuart K, Dedet JP, Blaineau C, Pagès $M$ : Mitotic stability of a coding DNA sequence-free version of Leishmania major chromosome I generated by targeted chromosome fragmentation. Gene 2002, 289:15I-9.

14. Martinez-Calvillo S, Yan S, Nguyen D, Fox M, Stuart KD, Myler PJ: Transcription of Leishmania major Friedlin chromosome I initiates in both directions within a single region. Mol Cell 2003, I I: I291-1299.

15. Noyes H, Pratlong F, Chance M, Ellis J, Lanotte G, Dedet JP: A previously unclassified trypanosomatid responsible for human cutaneous lesions in Martinique (French West Indies) is the most divergent member of the genus Leishmania s.s. Parasitology 2002, I 24:17-24.

16. Antequera F: Genomic specification and epigenetic regulation of eukaryotic DNA replication origins. EMBO J 2004, 23:4365-4370. 
17. Baker RE, Rogers K: Genetic and Genomic Analysis of theATRich Centromere DNA Element II of S. cerevisiae. Genetics 2005, I71:1463-1475.

18. Dubessay P, Blaineau C, Bastien P, Tasse L, Van Dijk J, Crobu L, Pagès $M$ : Cell cycle-dependent expression regulation by the proteasome pathway and characterization of the nuclear targeting signal of a Leishmania major Kin-13 kinesin. Mol Microbiol 2006, 59:1162-1174.

19. Makeyev AV, Liebhaber SA: The poly(C)-binding proteins: a multiplicity of functions and a search for mechanisms. RNA 2002, 8:265-278.

20. Tomonaga $T$, Levens $D$ : Activating transcription from single stranded DNA. Proc Natl Acad Sci USA 1996, 93:5830-5835.

21. Boisseau-Garsaud AM, Cales-Quist D, Desbois N, Jouannelle J, Jouannelle A, Pratlong F, Dedet JP: A new case of cutaneous infection by a presumed monoxenous trypanosomatid in the island of Martinique (French West Indies). Trans $R$ Soc Trop Med Hyg 2000, 94:5I-52

22. Thomaz-Soccol V, Pratlong F, Langue R, Castro E, Luz E, Dedet JP: New isolation of Leishmania enriettii Muniz andMedina, 1948 in Parana state, Brazil, 50 years after the firstdescription, and isoenzymatic polymorphism of the $L$. enriettii taxon. Ann Trop Med Parasitol I 996, 90:49|-495.

23. Stevens JR, Noyes HA, Schofield CJ, Gibson W: The molecular evolution of Trypanosomatidae. Adv Parasitol 200I, 48: I-56.

24. Rioux JA, Lanotte G, Serres E, Pratlong F, Bastien P, Périères J: Taxonomy of Leishmania. Use of isoenzymes. Suggestions for a new classification. Ann Parasitol Hum Comp 1990, 65: I I I- I 25.

25. EBI Site Index [http://www.ebi.ac.uk/services/]

26. Dr. Xuhua Xia's Webpage [http://dambe.bio.uottawa.ca/]

27. NCBI Homepage [http://www.ncbi.nlm.nih.gov/]

28. GeneDB [http://www.genedb.org/]

29. CBS Prediction Servers [http://www.cbs.dtu.dk/services/]

30. European Bioinformatics Institute [http://www.ebi.ac.uk/]

3I. Bangs JD, Uyetake L, Brickman MJ, Balber AE, Boothroyd JC: Molecular cloning and cellular localization of a BiP homologue in Trypanosoma brucei. Divergent ER retention signals in a lower eukaryote. J Cell Sci 1993, 105: I I0I-I3.

32. European Bioinformatics Institute Help Page [http:// www.ebi.ac.uk/clustalw/color frame.html]

Publish with Bio Med Central and every scientist can read your work free of charge

"BioMed Central will be the most significant development for disseminating the results of biomedical research in our lifetime. "

Sir Paul Nurse, Cancer Research UK

Your research papers will be:

- available free of charge to the entire biomedical community

- peer reviewed and published immediately upon acceptance

- cited in PubMed and archived on PubMed Central

- yours - you keep the copyright 